\title{
CONGENITAL INDIFFERENCE TO PAIN
}

Report of a Case

\author{
O. S. Ingwersen, NiJmegen, Holland \\ From the Orthopaedic Department of the Catholic University, Nijmegen
}

The world literature mentions only a few patients with congenital indifference to pain. Murray (1957) analysed thirty-one cases and Fanconi and Ferrazzini (1957) reviewed seventeen previously described cases. The following description concerns a patient observed in our department.

\section{CASE REPORT}

In 1962 a fifteen-year-old boy attended with a swollen left ankle with several discharging sinuses. Radiographs showed marked destruction of the joint suggestive of neuropathic arthritis (Fig. 1). This unusual finding in such a young person prompted further investigation. This revealed that when he was one year of age he had been treated by the paediatrician,

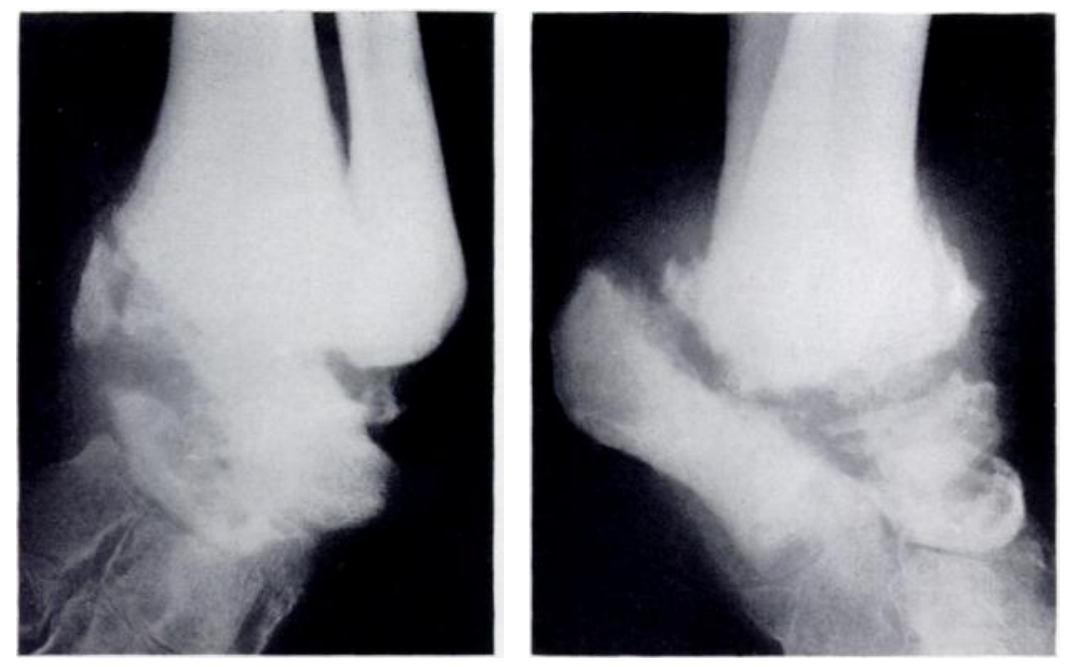

FIG. 1

Radiographic appearances of the left ankle in 1962.

Professor J. P. Slooff, for recurrent keratitis and inflammation of the tongue. Even at that age his teeth were loose in their sockets. The cornea was insensitive. There was generalised hypotonia of the skeletal muscles, deficient function of the superior oblique and lateral rectus muscles of the eyes, and marked laxity of the joints. Although a definite diagnosis was not made it was clear that this boy was suffering from some kind of generalised affection of the sensory system.

In the early years of his life he was treated repeatedly for corneal ulcers caused by scratching. A deep palmar burn occurred when he was two years old without causing evident pain. In this period the boy bit off the ends of several of his fingers.

When fourteen years of age he was seen by Professor G. M. San Giorgi on account of inflammation of the metatarso-phalangeal joint of the right foot. Radiographs showed damage to several joints of both feet, and admission to hospital was advised but was refused. The inflammation gradually subsided with conservative treatment and with special footwear. 
On his admission in 1962 we found a large-limbed, ungainly boy with many signs of degeneration. His brow was low, there was a low hairline and the eyebrows were continuous over the glabella (Fig. 2). The saddle nose was awry. The whole facial expression was somewhat suggestive of gargoylism. The mouth and the nostrils showed many fissures. The teeth were bad: several were missing and many showed serious decay. Scanty pubic and axillary hair, large penis, a tendency to obesity and many striae around the hips suggested an endocrine defect.

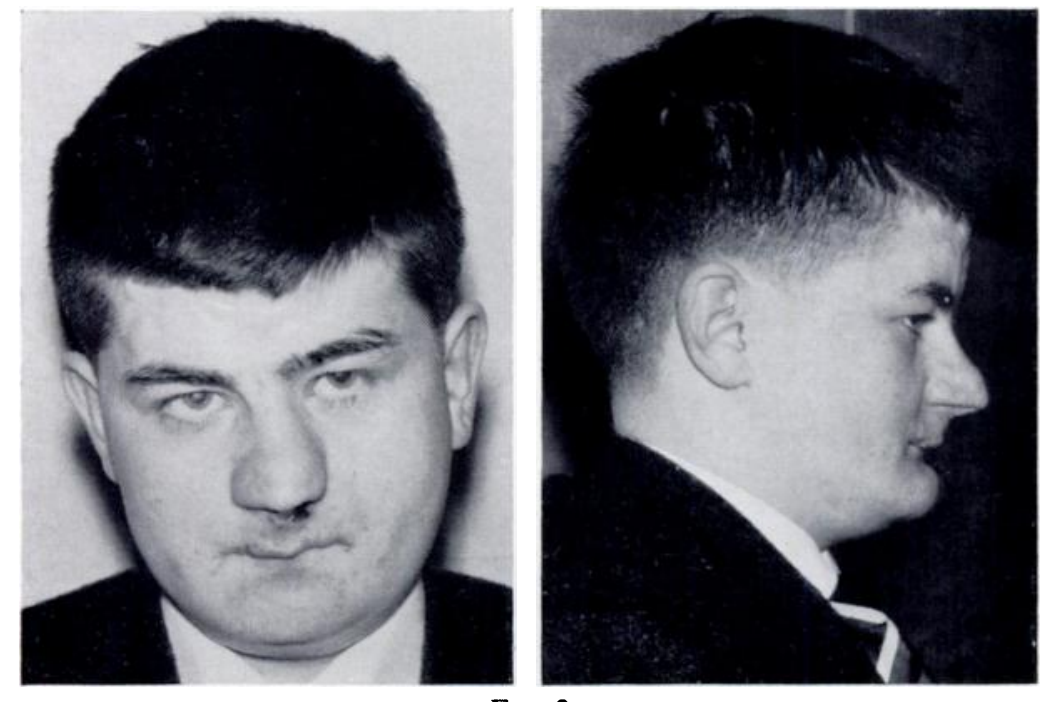

FIG. 2

The face at the time of admission. Note the low brow and hairline, and the distortion of the saddle nose.

Both hands were deformed and scarred: several fingers had the terminal segments missing (Figs. 3 to 5). The right foot showed talipes varus adductus of the Friedreich type. The left foot, especially the ankle region, was very swollen, suggesting the possibility of chronic inflammation, but there was no pain or redness. Hyperhidrosis was generalised.

Routine examination of blood and urine showed no abnormality.

Neurological findings-Neurological examination (Professor J. J. G. Prick) showed that there was a varied pattern of sensory perception. Definite hypalgesia was present over the left foot, the left wrist and the radial side of both hands. The same was found in the face above the eyebrows whereas complete analgesia was demonstrated over both corneae. The localisation test was accurate but in many places a needle could be stuck into the skin without causing pain though bleeding was produced. Sensibility to touch and deep perception were not impaired but the patient could not clearly define the difference between warmth and cold. Electrical examination did not show anything abnormal and the electro-encephalograph was undisturbed. The neurologist concluded that this was a case of definite degeneration without being able to determine whether the cause was central or peripheral. Leprosy was excluded (Professor J. W. H. Mali).

Other studies-Ophthalmological examination (Professor J. E. A. van den Heuvel) showed that the eyesight was impaired, the right eye showing $1 / 60$ and the left $5 / 60$. The absence of corneal sensitivity was confirmed and the several corneal scars were further examined. It was thought that these could be attributed to severe inflammation occurring shortly after birth.

Dental examination (Professor C. A. Merkx) showed that both jaws were very atrophic from recurring osteomyelitis and that the few remaining teeth were loose.

A psychological test (Dr P. B. Bierkens) showed that intelligence was somewhat below the average for his age, and that there were also very clearly difficulties of adaptation. On

VOL. 49 B, NO. 4, NOVEMBER 1967 
account of illness he was frequently away from school for long periods. His restricted eyesight and deformed hands partly prevented him from following normal instruction and made his acceptance in class rather difficult. In this respect he was hindered by his ambitious and frustrated mother who on several occasions prevented medical treatment. This was also apparent during his stay in hospital. Mostly the boy was easy going and compliant but very little was necessary to arouse and enrage him. He would not eat hot food but could not exactly explain why. He could not tolerate washing his scalp with warm water. He did not show the slightest insight into his malady.

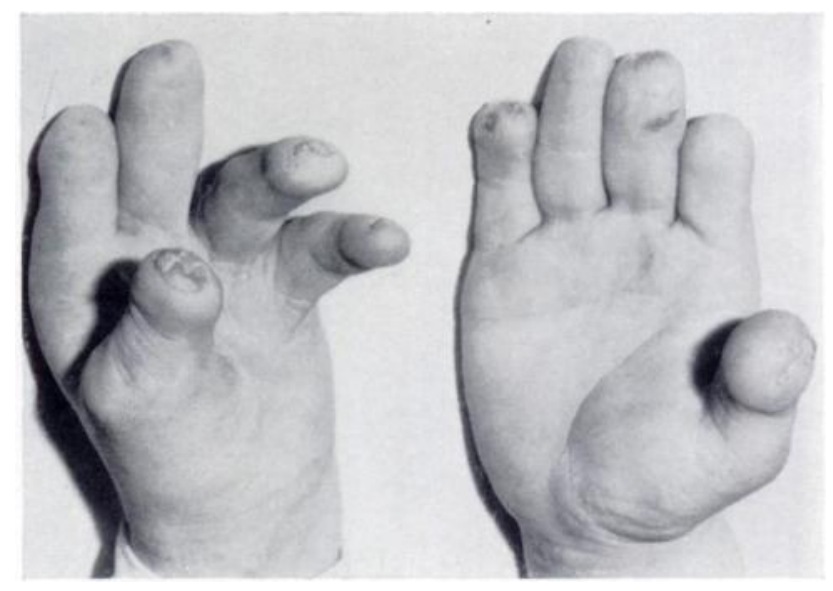

FIG. 3

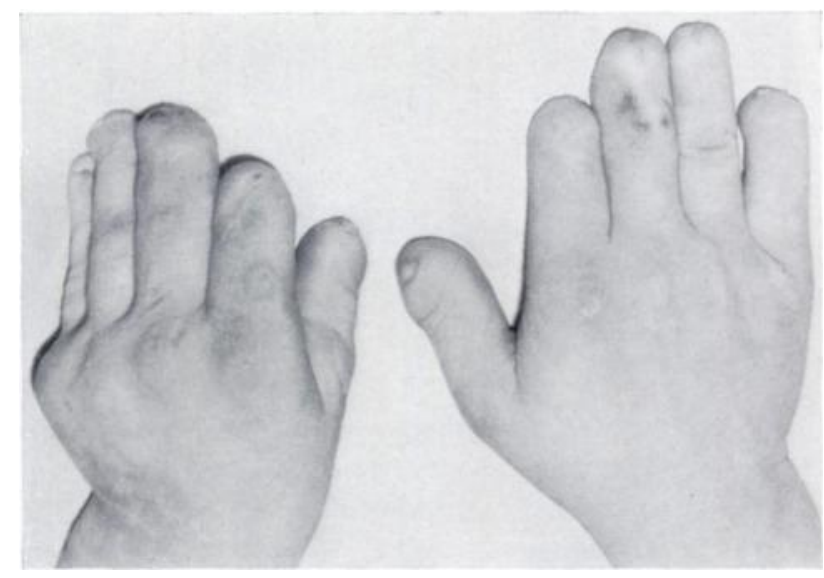

Fig. 4

The hands, showing the deformity and mutilation.

Radiographs showed that several joints were badly deformed (Figs. 6 and 7).

Treatment-Because of the obvious difficulties in the way of reconstructive surgery amputation was advised. This advice was accepted when there had been marked deterioration in the lad's general condition.

Both anaesthesia and operation were very well tolerated. The wound healed by first intention and sedatives were not necessary after operation despite some complaints of deep pain. When the boy was mobilised and was walking with crutches a simple transverse fracture of the left ulna with minor displacement occurred without any known cause (Fig. 8). Because 
of the vulnerability of the skin plaster-of-Paris was not applied. Even though callus formation was sufficient, in the long run the fracture did not heal and there is still a rigid pseudarthrosis which causes no pain (Figs. 9 and 10).

An artificial leg of the patellar tendon kearing type was very well tolerated for about three months. However, after a slight excoriation a deep phlegmonous inflammation developed. According to the patient the excoriation was caused by friction in the socket during a short walk, but it could have been caused by scratching. The boy is now under guidance in the department for rehabilitation.

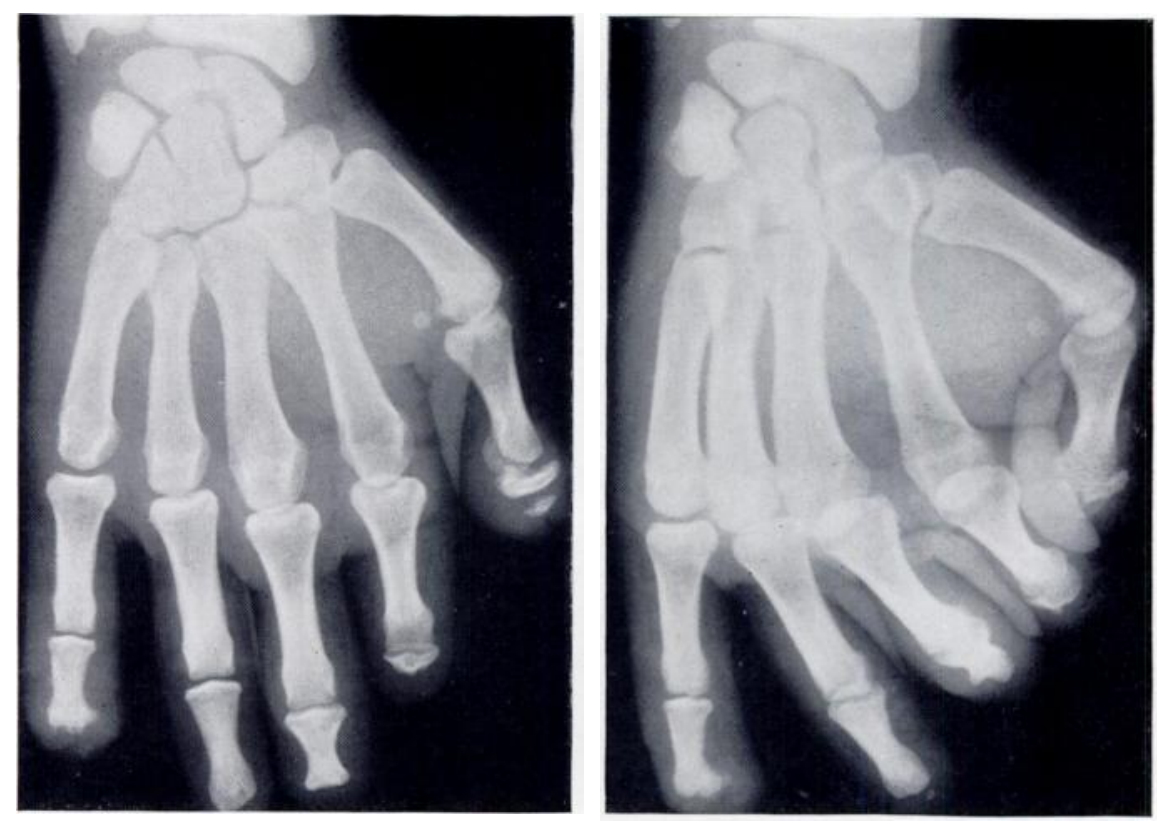

FIG. 5

Radiographic appearances of the hands.

Even though Fanconi thinks that prognosis is relatively good we feel that this may not be so in our case. The boy's demeanour shows that he is quite uninterested in his condition and in his ultimate fate. This, added to the fact that there is at least some mental retardation, makes it probable that we cannot count upon his full cooperation. He is being trained to use his prosthesis in the right way and to take the greatest possible care of his skin. It may be though that neglect of even a seemingly unimportant skin lesion may lead to severe inflammation with progressive loss of parts.

\section{DISCUSSION}

It is evident that this seemingly rare condition can easily be confused with acquired indifference to pain as described by Schilder in 1933 in a patient with defects of the supramarginal gyrus of the parietal lobe (Jewsbury 1951). The first case of true congenital indifference to pain was described in 1932 by Dearborn. Analysing thirty-one cases Murray (1957) pointed out some important aspects. For instance he mentioned that the joints resembled Charcot's joints and noted the recurring osteomyelitis and the proclivity to fractures. Other published reports are those of van der Houwen (1961) and Fischer (1965).

The most informative publication is Fanconi and Ferrazzini's (1957). They recapitulated the most important symptoms in these cases, all of which were present in our case: 1) lesions of the skin and mucous membranes; scars resulting from burns and biting the tongue, and self-mutilation of the fingers. 2) Deformities of the skeleton caused by spontaneous and 
traumatic fractures. 3) Loss of teeth caused by inflammation and abscess formation. 4) Deficiency of the sensory nervous system restricted to hypalgesia or analgesia, the other modalities being present. Analgesia of the cornea is present in many cases and the corneal reflex is often absent. As a rule aphasia as described in the acquired forms does not occur, nor do

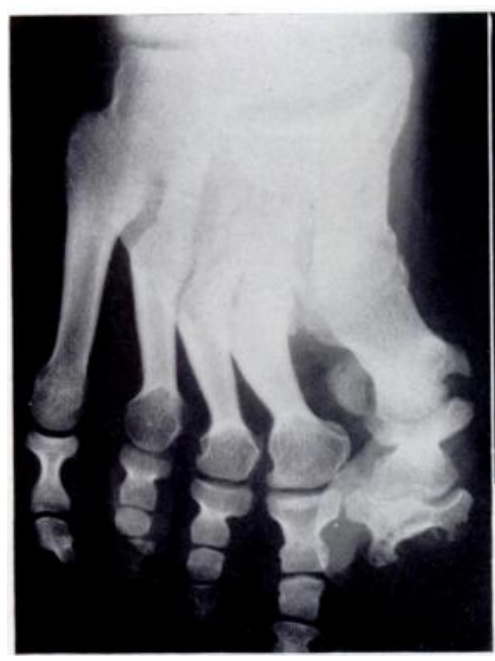

FIG. 6

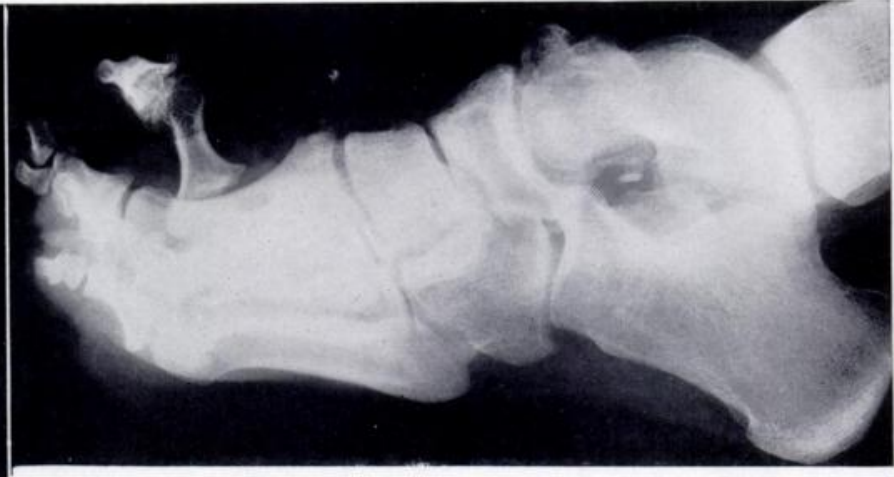

FIG. 7

FIGS. 6 AND 7

Radiographic appearances of the right foot. Note the destruction of the metatarso-phalangeal joint of the great toe.

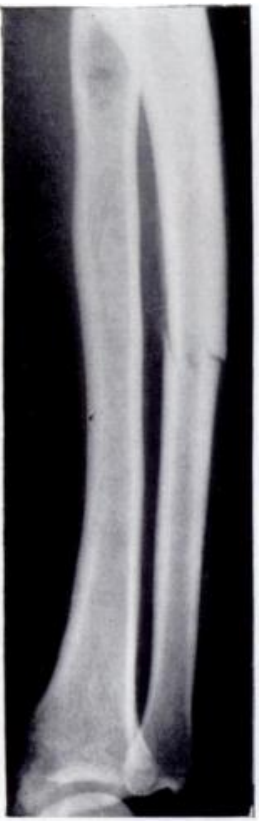

FIG. 8

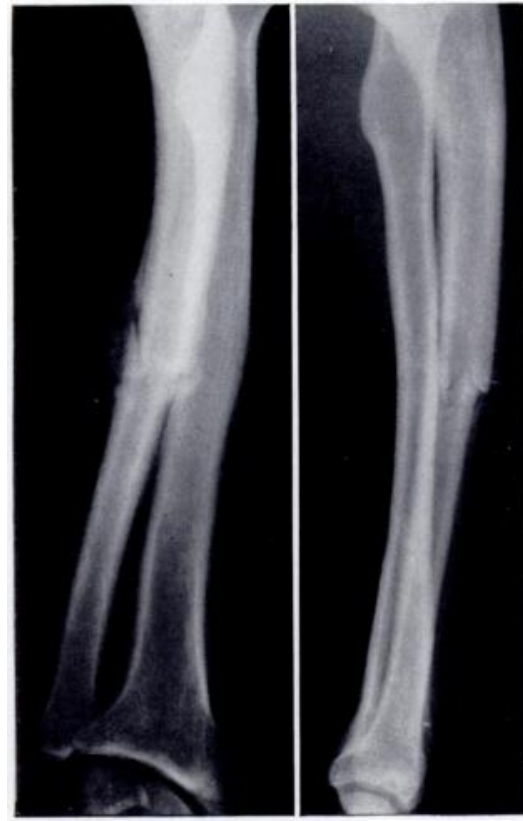

FIG. 9

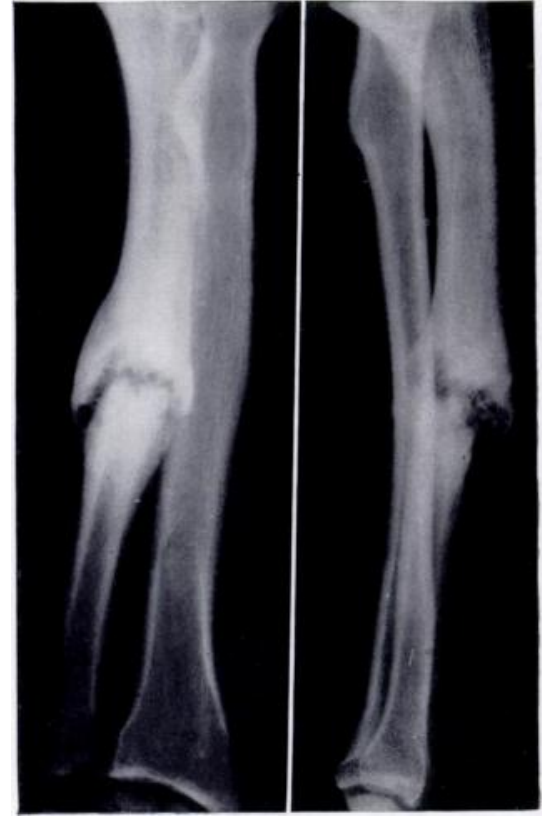

Fig. 10

The ulnar fracture. Figure 8-Fracture through the shaft of the left ulna. Figures 9 and $10-$ The fracture's progress to non-union. Early callus formation (Fig. 9). Established non-union (Fig. 10).

other neurological disturbances (Jéquier and Deller 1956). 5) Normal personality structure and normal intellectual functions. 6) No definite pathological pattern found in either bone (Petrie 1953) or skin. (Ortiz de Zarate (1955) found in one case that there were no free terminal endings of the nerves in the skin). 7) Prognosis seems to be good in most cases, probably because of the protection given by mature reason. 
Causation-No causative factor has been found, nor has the site of the primary defect been determined. It may be that the affection is caused by dysfunction of the hypothalamic region (Critchley 1956) but as far as we know research in this aspect has not yet been done. Nor is the exact mechanism of joint destruction very well understood; Harris and Brand's (1966) suggestion seems to be of interest.

In this respect it may be important that a girl from the same family suffered from the same affection in a more pronounced form. Seven other children from the same family (and presumably the whole generation) showed no abnormalities of this kind. Genealogical examination was not however performed. This affected girl died from phenobarbitone poisoning. A very thorough necropsy was done by Professor J. P. Slooff. Pathological, histological and histochemical methods failed to show any specific alterations in the peripheral or central nervous systems. As far as we know, congenital insensitivity to pain in two such close relations has not yet been described. Dyck, Kennel, Magal and Kraybill (1965) described hereditary sensory neuropathy but we feel that they described a completely different clinical picture. Even if Dyck's patients are similar in respect of congenital indifference to pain, they showed not only important changes in the feet such as talipes cavus with claw toes and atrophy of the muscles of the calf, but also ulcers and increase in diameter of the peripheral nerves. Differential diagnosis-Various neurological diseases and leprosy should be considered. On account of the manifold fractures the possibility of osteopsathyrosis should be excluded.

\section{SUMMARY}

1. A case of congenital indifference to pain in a boy aged fifteen years is described. The boy's sister was similarly affected.

2. Clinical features and treatment are described, and the features are compared with those in previously reported cases.

3. Causation is discussed in the light of the negative findings at necropsy on the patient's sister.

I wish to express my sincerest thanks to Professor G. M. San Giorgi and all those who have helped me in composing this paper and who are mentioned in the text.

\section{REFERENCES}

Critchley, M. (1956): Congenital Indifference to Pain. Annals of Internal Medicine, 45, 737.

Dearborn, G. Van N. (1932): A Case of Congenital General Pure Analgesia. Journal of Nervous and Mental Disease, 75, 612.

Dyck, P. J., Kennel, A. J., Magal, I. V., and Kraybill, E. N. (1965): A Virginia Kinship with Hereditary Sensory Neuropathy Peroneal Muscular Atrophy and Pes Cavus. Mayo Clinic Proceedings, 40, 685.

Fanconi, G., and Ferrazzini, F. (1957): Kongenitale Analgie, Kongenitale generalisierte Schmerzindifferenz. Helvetica Paediatrica Acta, 12, 79.

FISCHER, J. F. (1965): Arthropathie des Kniegelenkes bei kongenitaler Analgie. Zeitschrift für Orthopädie und ihre Grenzgebeite, 100, 489.

Harris, J. R., and Brand, P. W. (1966): Patterns of Disintegration of the Tarsus in the Anaesthetic Foot. Journal of Bone and Joint Surgery, 48-B, 4.

Houwen, H. van der (1961): A Case of Neuropathic Arthritis Caused by Indifference to Pain. Journal of Bone and Joint Surgery, 43-B, 314.

JÉquier, M., and Deller, M. (1956): L'indifférence congénitale à la douleur. Confinia Neurologica, 16, 207.

JewsBury, E. C. O. (1951): Insensitivity to Pain. Brain, 74, 336.

Murray, R. O. (1957): Congenital Indifference to Pain with Special Reference to Skeletal Changes. British Journal of Radiology, 30, 2.

Ortiz de Zarate, J. C. (1955): Analgésie généralisée congénitale. Encéphale, 44, 414.

Petrie, J. G. (1953): A Case of Progressive Joint Disorders Caused by Insensitivity to Pain. Journal of Bone and Joint Surgery, 35-B, 399.

SCHILDER, P. (1933): Quoted by Jewsbury, E. C. O. (1951).

VOL. $49 \mathrm{~B}$, NO. 4 , NOVEMBER 1967 